\title{
Follicle Stimulating Hormone Levels and Subclinical Atherosclerosis in Older Postmenopausal Women
}

\author{
L Niskanen, ER Bertone-Johnson, JK Virtanen, T Nurmi, J Mursu, S Voutilainen, K Ronkainen, \\ $J$ Kauhanen, TP Tuomainen \\ University of Massachusetts, Amherst, MA, USA; University of Eastern Finland, Kuopio, Finland; \\ Helsinki University Hospital, Helsinki, Finland.
}

\section{Introduction}

- Recent studies suggest that follicle stimulating hormone (FSH) may play a role in cardiovascular health, independent of estradiol (E2) ${ }^{1}$

- Previous studies have evaluted FSH and atherosclerosis in women during the menopausal transition, when levels are highly dynamic

- Whether FSH after menopause is associated with aterosclerosis is unknown.

\section{Objectives}

- We assessed the relation of postmenopausal FSH and E2 levels with carotid artery intima-media thickness (IMT), a measure of atherosclerosis.

- Study participants were members of the Kuopio Ischaemic Heart Disease (KIHD) Risk Factor Study, a prospective study of risk factors for cardiovascular disease in men and women in eastern Finland.

\section{Methods}

- Participants were 588 female KIHD members, aged 53-73 at baseline (1998-2001) and not using hormone therapy (HT).

- Women completed a clinical examination, during which common carotid artery (CCA) IMT was measured via high resolution Bmode ultrasonography. Mean IMT was averaged from 100 measures of the left and 100 of the right CCA. ${ }^{2}$

- Fasting blood samples were assayed for FSH and E2, along with testosterone, plasma lipids, insulin and glucose.

- Participants self-reported data on demographics, behavioral, reproductive and health factors via questionnaire.

- Height and weight were directly measured, and study physicians recorded prevalent cardiovascular and metabolic disease.

- Multivariable linear regression was used to assess relations of FSH and E2 with mean IMT.

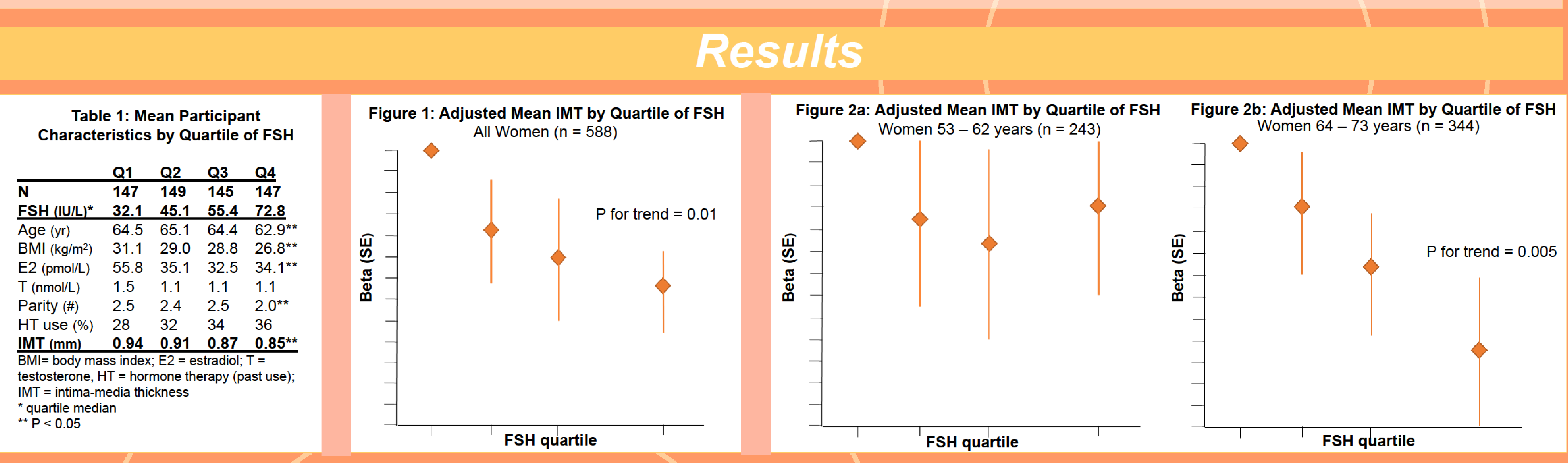

- Mean IMT in women in quartiles $1-4$ of FSH were $0.94,0.91,0.87$ and $0.85 \mathrm{~mm}$, respectively $\left(P_{\text {trend }}<0.001 ;\right.$ Table 1$)$.

- FSH levels remained inversely associated with IMT in multivariable models $\left(P_{\text {trend }}=0.01\right.$; Figure 1$)$.

- Models were adjusted for age, enrollment year, E2, body mass index, waist to hip ratio, physical activity, past HT use, duration of HT use, smoking status, pack-years of smoking alcohol use, parity, age at menopause, systolic \& diastolic blood pressure, testosterone, lipid levels (total, LDL, \& HDL cholesterol \& triglycerides), fasting glucose, fasting insulin, history of hypertension, diabetes, symptomatic heart disease and stroke, and current use of medications for hypertension, diabetes, high cholesterol and heart disease.

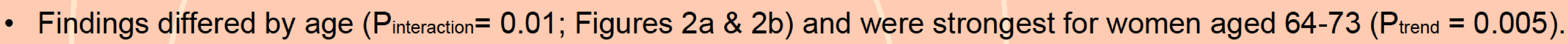

- In contrast, E2 levels were unrelated to IMT $(P>0.05)$. Additionally, findings did not vary by body mass index $(P$ interaction $>0.05)$.

\section{Conclusions}

- Postmenopausal FSH levels were significantly and inversely associated with common carotid artery intima-media thickness.

- Relations were strongest among older postmenopausal women (ages 64-73).

- Associations of FSH and IMT were not explained by confounding or effect modification by adiposity, estradiol levels, or prevalent cardiometabolic disorders.

- Prospective studies of postmenopausal FSH and atherosclerosis progression are warranted to better understand these relations and clarify potential underlying physiology.

1) EI Khoudary SR, Santoro N, Chen HY, Tepper PG, Brooks MM, Thurston RC, Janssen I, Harlow SD, Barinas-Mitchell E, Selzer F, Derby CA, Jackson EA, McConnell D, Matthews KA. Trajectories of estradiol and follicle-stimulating hormone over the menopausal transition and early markers of atherosclerosis after menopause. Eur J Prev Cardiol. 2015: doi:10.1177/2047487315607044

2) Salonen JT, Seppänen K, Lakka TA, Salonen R, Kaplan GA. Mercury accumulation and accelerated progression of carotid atherosclerosis: a population-based prospective 4-year follow-up study in men in eastern Finland. Atherosclerosis. 2000:148:265-273. 